\title{
Microbial infections in patients with congenital pulmonary airway malformation
}

\author{
O. Voronina', N. Ryzhova', M. Kunda'1, N. Sharapova', E. Aksenova', M. Egorov², A. Ignatova², Y. \\ Gorinova', O. Simonova', A. Gintsburg'.
}

1 - N.F. Gamaleya National Research Center of Epidemiology and Microbiology, Ministry of Health of Russia, Moscow, Russia.

2 - National Medical Research Center of Children's Health, Ministry of Health of Russia, Moscow, Russia.

Keywords: congenital pulmonary airway malformation; microbial infection; molecular-genetic methods; Proteobacteria.

Background. Cystic Fibrosis (CF) and primary ciliary dyskinesia (PCD) are the most famous autosomal recessive disorder associated with impaired mucociliary clearance, which promotes upper and lower airway infection. Comparison of CF and PCD airways microbiota is traditional approach in the analysis. In contrast we had possibility to compare PCD with other diseases in the group of congenital pulmonary airway malformation (CPAM). The present study aimed to reveal characteristics of the Proteobacteria airway infection in different CPAM diseases.

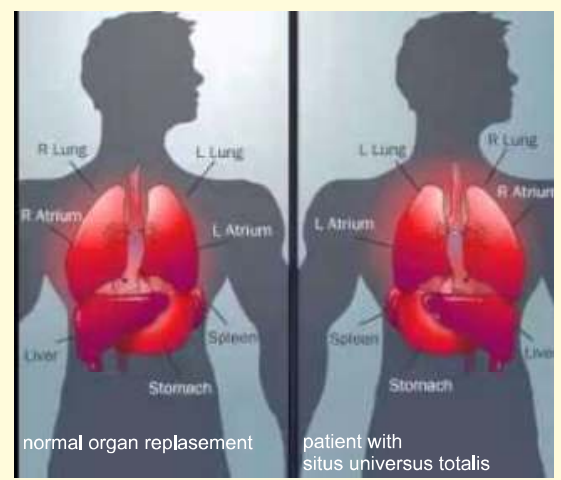

Fig. 1. Kartagener syndrome. https://youtu.be/T2LaVi4h4nc

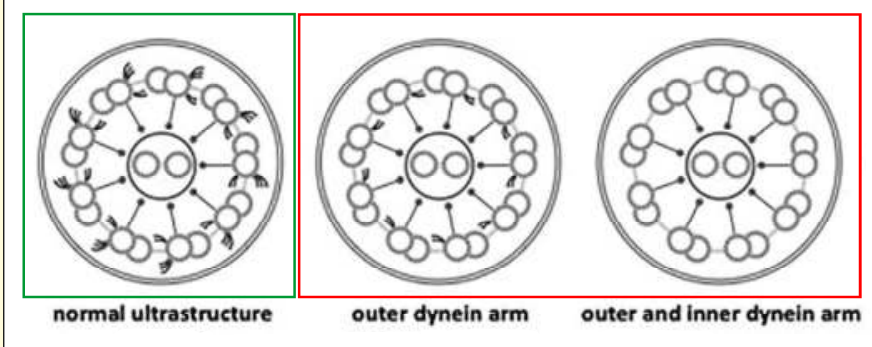

Fig.2. Classification of genes mutated in primary ciliary dyskinesia segregated based on characteristic ultrastructural defects of the axoneme. Ferkol T. Movement. Paediatr Respir Rev. 2017, 24:19-20. doi: 10.1016/j.prrv.2017.06.005.

Results. Only two PCD patients $(11 \%)$ were infected by Gammaproteobacteria (Haemophilus influenzae and Pseudomonas aeruginosa), and one patient was infected by Burkholderia cenocepacia ST878 (Betaproteobacteria). These three patients of 9-15 year old had pulmonary disorder complicated by otitis and rhinosinusitis. $83 \%$ of PCD children (1-18 years old) were infected by Lautropia mirabilis (Burkholderiaceae). In the group of other CPAM diseases there were $42 \%$ of children (1-16 years old) infected by Gammaproteobacteria in association with Fungi, and $38 \%$ of patients were infected by $L$. mirabilis.
Methods. Tracheal aspirate and sputum of 18 children with PCD and 24 children with other CPAM diseases were analyzed by molecular-genetic methods on the base of amplification and sequencing of microbial ribosomal and housekeeping genes. The children of 1-18 years old were hospitalized in the department of pulmonology and allergology of National Medical Research Center of Children's Health.

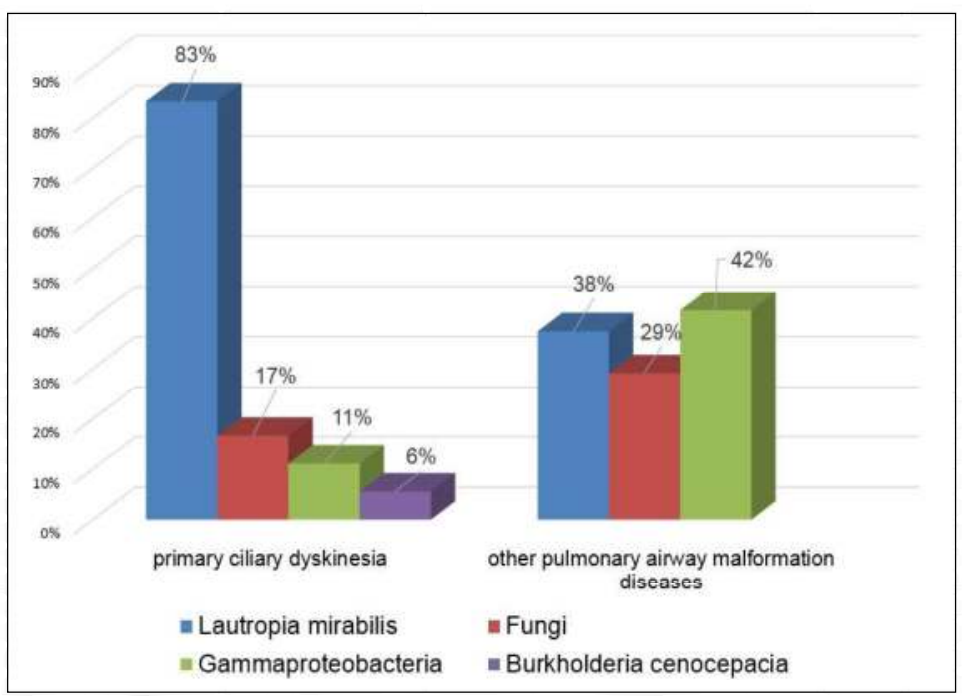

Fig. 3. Characteristics of the Proteobacteria airway infection in different pulmonary airway malformation diseases.

Conclusions. Important differences in airway microbiology between PCD and other CPAM were revealed. L. mirabilis could be considered the early marker of chronic lung disorder. 